\title{
Relationship Between Behavioral and Physiological Spectral-Ripple Discrimination
}

\author{
Jong Ho Won ${ }^{1,2,3}$, Christopher G. Chinard ${ }^{3,4}$, Seeyoun Kwon ${ }^{5,6}$, Vasant K. Dasika ${ }^{1}$, Kaibao Nie ${ }^{1}$, \\ Ward R. Drennan ${ }^{1}$, Kelly L. Tremblay ${ }^{3}$, and Jay T. Rubinstein ${ }^{1,2}$ \\ ${ }^{1}$ Virginia Merrill Bloedel Hearing Research Center, Department of Otolaryngology-Head and Neck Surgery, University of \\ Washington, Seattle, WA 98195, USA \\ ${ }^{2}$ Department of Bioengineering, University of Washington, Seattle, WA 98195, USA \\ ${ }^{3}$ Department of Speech and Hearing Sciences, University of Washington, Seattle, WA 98195, USA \\ ${ }^{4}$ Department of Communication Sciences and Disorders, James Madison University, Harrisonburg, VA 22807, USA \\ ${ }^{5}$ Department of Otolaryngology-Head and Neck Surgery, Samsung Medical Center, Seoul 135-710, South Korea \\ ${ }^{6}$ Department of Biomedical Engineering, Hanyang University, Seoul 133-791, South Korea
}

Received: 6 October 2010; Accepted: 12 January 2011; Online publication: 27 January 2011

\begin{abstract}
Previous studies have found a significant correlation between spectral-ripple discrimination and speech and music perception in cochlear implant (CI) users. This relationship could be of use to clinicians and scientists who are interested in using spectral-ripple stimuli in the assessment and habilitation of CI users. However, previous psychoacoustic tasks used to assess spectral discrimination are not suitable for all populations, and it would be beneficial to develop methods that could be used to test all age ranges, including pediatric implant users. Additionally, it is important to understand how ripple stimuli are processed in the central auditory system and how their neural representation contributes to behavioral performance. For this reason, we developed a singleinterval, yes/no paradigm that could potentially be used both behaviorally and electrophysiologically to
\end{abstract}

\footnotetext{
This paper was a part of the first author's PhD dissertation. Portions of this data were presented at the 2007 Northwest Auditory and Vestibular Meeting at Portland, OR, USA at the 2008 ARO Midwinter Meeting at Phoenix, AZ, USA at the 2008 American Auditory Society Annual Meeting at Scottsdale, AZ, USA, and at the 2011 ARO Midwinter Meeting at Baltimore, MD, USA.
}

Correspondence to: Jong Ho Won · Virginia Merrill Bloedel Hearing Research Center, Department of Otolaryngology-Head and Neck Surgery · University of Washington - Seattle, WA 98195, USA. Telephone: +1-206-6162041; fax: +1-206-6161828; email: jhwon@uw. edu estimate spectral-ripple threshold. In experiment 1 , behavioral thresholds obtained using the single-interval method were compared to thresholds obtained using a previously established three-alternative forcedchoice method. A significant correlation was found $(r=0.84, p=0.0002)$ in 14 adult CI users. The spectralripple threshold obtained using the new method also correlated with speech perception in quiet and noise. In experiment 2 , the effect of the number of vocoderprocessing channels on the behavioral and physiological threshold in normal-hearing listeners was determined. Behavioral thresholds, using the new singleinterval method, as well as cortical P1-N1-P2 responses changed as a function of the number of channels. Better behavioral and physiological performance (i.e., better discrimination ability at higher ripple densities) was observed as more channels added. In experiment 3 , the relationship between behavioral and physiological data was examined. Amplitudes of the P1-N1-P2 "change" responses were significantly correlated with $d^{\prime}$ values from the single-interval behavioral procedure. Results suggest that the single-interval procedure with spectral-ripple phase inversion in ongoing stimuli is a valid approach for measuring behavioral or physiological spectral resolution.

Keywords: spectral-ripple discrimination, behavioral and physiological measure, cochlear implant, auditory change response, auditory cortex 


\section{INTRODUCTION}

Spectral-ripple stimuli consist of rippled spectra noise signals in which the frequency positions of the spectral peaks and valleys alternate (Supin et al. 1994). Over the past few years, spectral-ripple discrimination has been widely used in a variety of behavioral experiments with normal-hearing $(\mathrm{NH})$, hearingimpaired (HI), and cochlear-implant (CI) listeners (e.g., Supin et al. 1994; Henry et al. 2000, 2005; Won et al. 2007; Litvak et al. 2007; Saoji et al. 2009; Won et al. 2010). For example, the ability of subjects to discriminate a reversal in the phase of the rippled shape (Henry et al. 2000, 2005; Won et al. 2007), and the ability to differentiate between a spectral-ripple stimulus and white noise (Litvak et al. 2007) have been evaluated. Furthermore, spectral-ripple discrimination ability has been shown to correlate significantly with vowel and consonant recognition in quiet (Henry et al. 2005; Litvak et al. 2007), word recognition in noise (Won et al. 2007), and music perception abilities including complex-tone pitch direction discrimination, melody recognition, and timbre recognition (Won et al. 2010) for CI users. Drennan et al. (2010) used spectral-ripple discrimination to evaluate different CI sound encoding strategies, and Faulkner et al. (2010) used spectralripple discrimination for auditory training in CI listeners to improve the perception of spectral details in speech and music sounds. Because Won et al. (2007) showed that test-retest reliability for the spectral-ripple test was good, and with minimal learning effects, the spectral-ripple test appears to be a stable measure that could be used in research as well as the clinic. These previous studies suggest that spectral-ripple discrimination is an efficient and valuable tool for assessing spectral envelope sensitivity in CI users in a way that can predict the hearing performance.

An additional benefit of spectral-ripple testing is that it is a non-linguistic psychophysical measure that could be helpful to researchers and clinicians when testing infants and children. Such information could potentially be used to improve CI signal processing or (re)habilitation strategies. However, the multipleinterval, adaptive forced-choice (AFC) discrimination procedures that have been typically used with adult listeners (e.g., three interval, three-AFC procedure) are poorly suited for behavioral studies with infants and toddlers. The unchanging stimuli of a multipleinterval procedure are also unsuitable for measuring spectral-ripple discrimination electrophysiologically via auditory evoked potentials. For these reasons, the goal of the present study was to develop a new method of measuring spectral-ripple threshold. The new method uses a single-interval, yes/no paradigm with the method of constant stimuli. On a trial, either a "change" or a "no-change" stimulus was presented. The stimulus paradigm and physiological analysis techniques were modeled after those used by Ross et al. (2007). This single-interval method can be extended with minimal changes to test preverbal children using a conditioned head turn or observerbased procedure (e.g., Eisenberg et al. 2007; Dasika et al. 2009). Moreover, this type of stimulus presentation is also useful because the same stimuli and presentation mode used for behavioral perceptual testing are also feasible for studying the neural detection of spectral changes.

Electrophysiological measures have long been used to assess the integrity of the auditory system following implantation (for review, see Abbas and Brown 2000; Martin et al. 2008) as well as to assist with CI mapping (Brown et al. 2000). Electrophysiological measures can be especially helpful in assessing the integrity of the auditory system in infants and children when behavioral testing is challenging or not possible. For these reasons, the present study attempted to determine if electrophysiological responses could be evoked by spectral-ripple stimuli, and estimated the physiological capacity of an individual's auditory system in detecting spectral changes within spectralripple stimuli. The P1-N1-P2 complex, a cortical auditory evoked potential, is especially relevant for this purpose because the response is robust enough to reliably identify in individuals. The P1-N1-P2 response may be elicited by the onset or offset of, or changes within, an acoustic stimulus (Spoor et al. 1969; Jerger and Jerger 1970; McCandless and Rose 1970). These P1-N1-P2 responses provide information about sound processing up to the level of the cortex, and can closely approximate behavioral discrimination thresholds (for review: Martin et al. 2008). For this reason, cortical auditory evoked potentials have been used extensively with CI listeners in response to pulse trains (Ponton et al. 1996) or speech sounds (Friesen and Tremblay 2006), as well as vocoded speech in normalhearing listeners (Friesen et al. 2009).

The goals of the current study are (1) to determine if the previously used three-AFC procedure yielded results that were correlated with the new, singleinterval, yes/no procedure; (2) to determine the feasibility of recording cortical P1-N1-P2 responses using the spectral-ripple stimuli; and (3) to determine if these physiological responses are related to behavioral spectral-ripple discrimination. In experiment 1 , the proposed single-interval, yes/no procedure with the method of constant stimuli was conducted with adult CI listeners. Results were compared to data from the previously established spectral-ripple discrimination test which used a three-interval, three-AFC, adaptive procedure (e.g., Won et al. 2007). In experi- 
ment 2, the single-interval, yes/no spectral-ripple discrimination procedure was used to obtain behavioral and physiological measures in NH listeners. The effect of the number of vocoder-processing channels on behavioral thresholds and the P1-N1-P2 responses were examined. In experiment 3 , the correlation between $d^{\prime}$ values from the single-interval behavioral performance and the amplitudes of P1-N1-P2 change responses were examined.

\section{METHODS}

Experiment 1: the development and validation of single-interval spectral-ripple discrimination

Subjects. Fourteen post-lingually deafened, generally high performing, adult CI users participated in experiment 1 . They were $25-77$ years old (mean= 57 years, six females and eight males). Individual CI subject information is listed in Table 1 . All subjects listened to the stimuli using their own sound processor set to a comfortable listening level. CI sound processor settings were fixed so that sound was processed identically throughout all test batteries. The use of human subjects in this study was reviewed and approved by the University of Washington Institutional Review Board.

Stimuli and procedures.

1. The single-interval, yes/no paradigm spectralripple discrimination test

Four hundred pure-tone frequency components were summed to generate the rippled noise stimuli. Amplitudes of the components were determined by a full wave-rectified sinusoidal envelope on a logarithmic amplitude scale. The 400 tones were spaced equally on a logarithmic frequency scale. The ripple peaks were spaced equally on a logarithmic frequency scale. Bandwidth of the stimuli was $100-5,000 \mathrm{~Hz}$. The peakto-valley ratio of the ripple spectra was $30 \mathrm{~dB}$. Standard and inverted (ripple phase reversed) ripple stimuli were generated. For standard ripples, the phase of the full wave-rectified sinusoidal spectral envelope was set to zero radians; and for inverted ripples, it was set to $\pi / 2$. Using standard and inverted ripples, two types of test stimuli were generated: the "standard-standard" ripple and the "standardinverted" ripple.

For the standard-inverted case (i.e., the change stimulus), the first $2 \mathrm{~s}$ consisted of a standard ripple and the last $2 \mathrm{~s}$ contained an inverted ripple. Therefore, there was a spectral change at 2-s point. Each half of each stimulus was individually created and then concatenated. To eliminate the possibility of a loudness change at the 2-s point of the standardinverted stimuli, the root mean square of the first (standard ripple) and the last (inverted ripple) parts were matched. After amplitude matching, the whole 4-s stimulus was ramped with $150-\mathrm{ms}$ rise/fall times, and filtered with a long-term, speech-shaped filter (Byrne et al. 1994). Because the concatenation could produce frequency components beyond $5 \mathrm{kHz}$ at the 2-s point, a $5 \mathrm{kHz}$ low-pass filter was applied to the entire 4-s stimuli. To prevent a subject from perceiving a cue due to the temporal pattern of transition from standard to inverted ripple at the 2-s point, the phase of both the first and last $2 \mathrm{~s}$ was randomized for every presentation using random phases for the 400 individual frequency components.

For the standard-standard case (i.e., no-change stimulus), a 4-s duration standard ripple stimulus was created and ramped with 150-ms rise/fall times, then filtered with a long-term, speech-shaped filter (Byrne et al. 1994) and with a $5 \mathrm{kHz}$ low-pass filter. Therefore, there was no change within the stimulus.

Figure 1 shows waveforms (upper panels) and spectrograms (middle panels) for the standardinverted and standard-standard stimuli for the 1 ripple/octave case. The bottom row of Fig. 1 shows example waveforms for the standard-inverted and standard-standard stimuli from 1.98 to $2.02 \mathrm{~s}$, showing that there is no temporal discontinuity cue, such as a sudden amplitude change, for a subject to use to detect at the midpoint of the standard-inverted stimuli. There is therefore only a spectral cue that a subject can use to detect a change within the standardinverted stimuli.

Figure 2 shows the CI sound processor output in response to the standard-inverted ripple stimuli using HiResolution strategy. The upper two plots show how HiResolution strategy encodes the standard-inverted ripple stimuli over the duration of stimuli. The left panel shows the output for spectral-ripple density of 1.0 ripple/octave, and the right panel shows the output for ripple density of 6.0 ripples/octave. The lower panel shows average outputs over the first $2 \mathrm{~s}$ (i.e., standard phase) and the last $2 \mathrm{~s}$ (i.e., inverted phase) of the standard-inverted stimuli. The upper left panel (1 ripple/octave) shows that there is a distinct change at the 2-s point for all electrodes, but the upper right panel (6 ripples/octave) does not show visible change at the 2-s point in the outputs for all 16 electrodes. The lower panel plots also confirm this trend. Multiple peaks and valleys are wellrepresented across the electrodes when ripple density is low. But at higher ripple density, the distance between the peaks and valleys is decreased, so the sound processor presents reduced spectral contrast for higher ripple density.

A single administration of the single-interval, yes/ no paradigm spectral-ripple discrimination task involved four blocks of test trials. Sounds were 


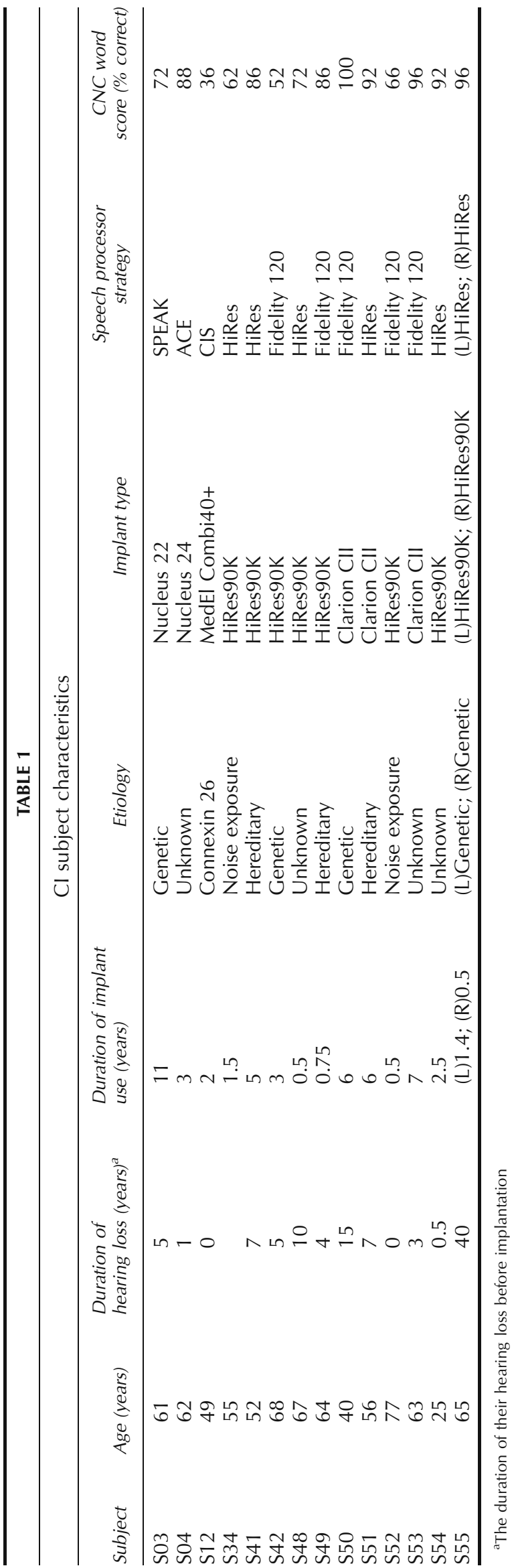

presented at $65 \mathrm{dBA}$ in the free field in a soundtreated booth via a single loudspeaker, positioned $1 \mathrm{~m}$ from the subject. Ripple stimuli were generated with 15 different densities: $0.25,0.5,0.707,1,1.414,2$, $2.828,4,5.657,8,11.314,16,22.627,32$, and 40 ripples/octave. The method of constant stimuli was used. Ten consecutive ripple densities from 0.25 to 8 ripples/octave were tested; however, if a subject could discriminate 8 ripples/octave stimuli above chance levels, higher ripple densities were tested. Each ripple density was presented 10 times in random order within a block: five times for standard-standard ripple stimuli and another five times for standardinverted ripple stimuli. Therefore, a total of 20 standard-standard and standard-inverted ripple stimuli were presented across the four test blocks. Subjects performed the task using a mouse and computer screen placed in front of and to the right of them. Subjects were instructed to listen to the sound and click on an on-screen button labeled "Yes" if they thought there was a change within the stimulus; or "No" if they thought there was not a change. Percent correct and hit rate were calculated for each block. Feedback was not provided. The testing required $1 \mathrm{~h}$ for each subject.

Threshold was defined as the ripple density corresponding to $d^{\prime}=1$. Data from both Won et al. (2007) and the present study suggest that psychometric functions for spectral-ripple discrimination generally follow an expected reverse-sigmoid shape as a function of increasing ripple density. It was desirable to accurately estimate threshold from points statistically shown to lie on the slope of the psychometric function, rather than points on either the upper or lower asymptote. Like several previous studies, confidence interval analyses were used to identify points lying on the upper asymptote, slope, and lower asymptote of the psychometric function (e.g., Buss et al. 1986; Bargones et al. 1995; Dasika et al. 2009). At each ripple density, $d^{\prime}$ and $80 \%$ confidence intervals for $d^{\prime}$ were computed from aggregate hit and false alarm rates obtained from the four blocks. To avoid undefined values of $d^{\prime}$, hit rates of 0 were converted to $0.5 / \mathrm{N}_{\mathrm{s}}$ and hit rates of 1 were converted to $1-0.5 / \mathrm{N}_{\mathrm{s}}$, where $\mathrm{N}_{\mathrm{s}}$ was the number of standard-inverted stimuli presented at a given ripple density (i.e., 20; cf. Macmillan and Creelman 2005). Hit rate proportions of 1 or 0 were thus converted to 0.975 or 0.025 . Similarly, false alarm rates of 0 or 1 were converted to 0.025 or 0.975 . Eighty percent confidence intervals for $d^{\prime}$ were estimated by using the approximation described by Gourevitch and Galanter (1967; cf. Macmillan and Creelman 2005). Eighty percent as opposed to $95 \%$ confidence intervals were used to identify a greater number of data points on the slope. 

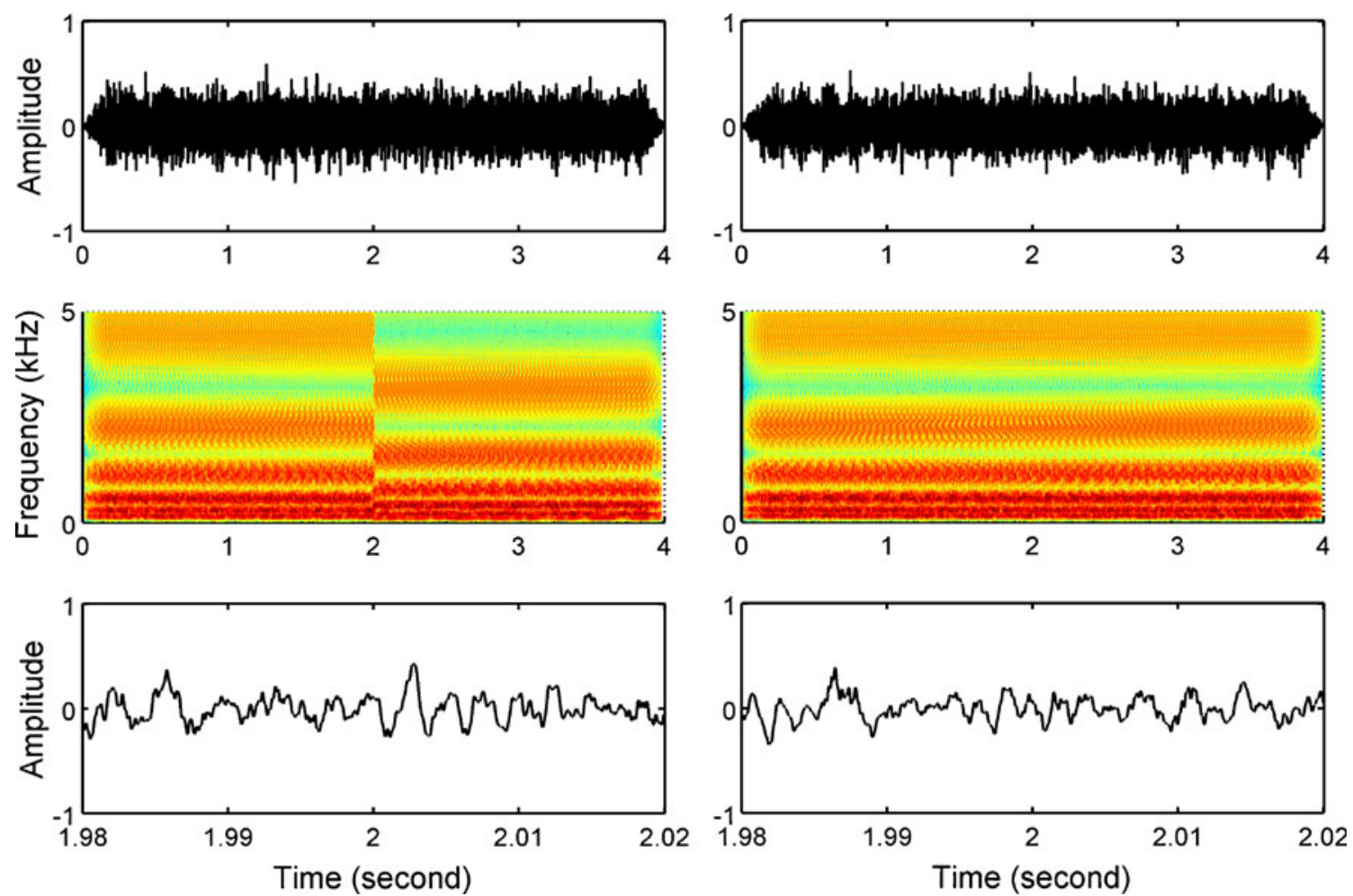

FIG. 1. Stimuli waveforms (upper panels), spectrograms (middle panels), and zoom-in of time-domain waveforms from 1.98 to $2.02 \mathrm{~s}$ (lower panels) for a standard-inverted stimulus (left side) and a standard-standard stimulus (right side). The ripple density was 1 ripple/octave for both stimuli.

The upper asymptote was taken as the maximum performance obtained at any ripple density. If the upper limit of the $80 \%$ confidence interval of $d^{\prime}$ of a given data point equaled or exceeded asymptotic $d^{\prime}$, the data point was generally assumed to lie on the upper asymptote. If the lower limit of the $80 \%$ confidence interval of $d^{\prime}$ for a given data point was lower than $d^{\prime}=0$ (chance performance), the point was generally assumed to lie on the lower asymptote. Slope was estimated using all successive data points with upper and lower confidence intervals contained entirely within the interval between the upper and lower asymptotes. Because few points were sometimes identified on the slope, the upper asymptotic point corresponding to the highest ripple density and the lower asymptotic point corresponding to the lowest ripple density were also included in the slope calculation. Sensitivity $\left(d^{\prime}\right)$ versus ripple density (ripples/ octave) data was fit by linear regression. Threshold was defined as the ripple density at which the regression line equaled 1 (i.e., $d^{\prime}=1$ ).

2. The three-interval, three adaptive forced-choice (3-AFC) spectral-ripple discrimination test

The three-interval, 3-AFC procedure used the same stimuli described by Won et al. (2007). Stimuli of $500 \mathrm{~ms}$ duration were generated by summing 200 pure-tone frequency components. Each 500-ms stim- ulus was either a standard or inverted ripple. The ripple densities included $0.125,0.176,0.250,0.354$, $0.500,0.707,1.000,1.414,2.000,2.828,4.000,5.657$, 8.000 , and 11.314 ripples/octave. The spectral-ripple resolution threshold was determined using a two-up, one-down adaptive procedure, converging on $70.7 \%$ correct (Levitt 1971). Each test run began with 0.176 ripples/octave and moved in equal ratio steps of 1.414. The presentation level was roved within trials ( $7 \mathrm{~dB}$ range in $1 \mathrm{~dB}$ steps). Subjects were asked to click on an on-screen button that was labeled 1, 2, and 3 after the stimuli were presented. One stimulus (i.e., inverted ripple sound, test stimulus) was different from two others (i.e., standard ripple sound, reference stimulus). The subject's task was to identify the test stimulus. Threshold for a single test run was estimated by averaging the final eight of 13 reversals. The primary dependent variable was determined by averaging the threshold from six different test runs. Feedback was not provided. The testing took $30 \mathrm{~min}$ for each subject.

\section{Speech reception threshold in noise test}

The procedure for administering the speech reception threshold (SRT) test was the same as that previously described by Won et al. (2007), and similar to Turner et al. (2004). Subjects were asked to identify one randomly chosen spondee word out of a closedset of 12 equally difficult spondees (Harris 1991). The 

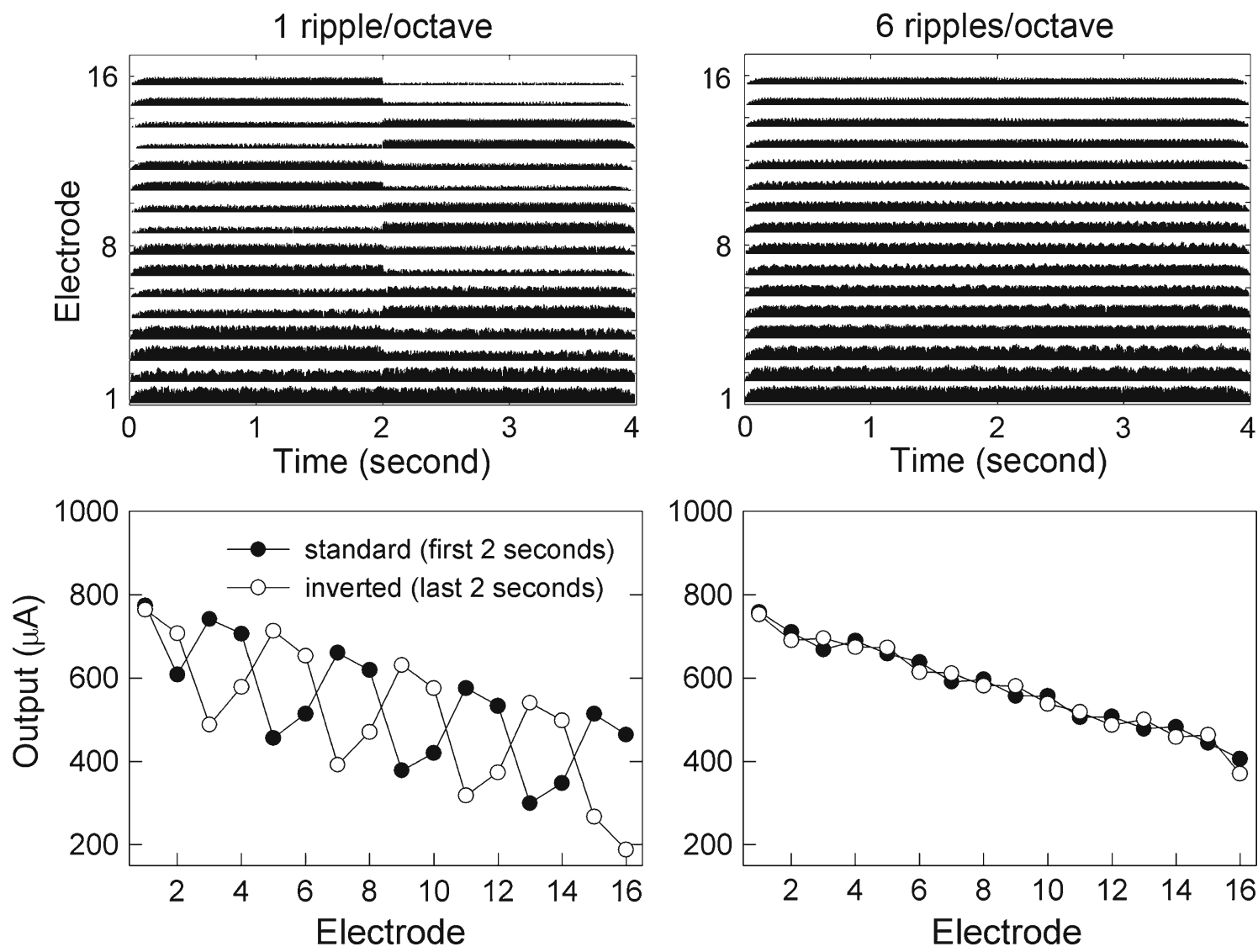

FIG. 2. The sound processor outputs for spectral-ripple density of 1 ripple/octave (left panel) and 6 ripples/octave (right panel) are shown. The upper panel plots show electrodograms for the standard-mixed ripple stimuli, which represent the biphasic pulses (in $\mu \mathrm{A}$ ) computed by

HiResolution strategy of Advanced Bionics devices. The lower panel plots show average outputs (in $\mu \mathrm{A}$ ) over the duration of the standard phase (first $2 \mathrm{~s}$, filled circles) and the inverted phase (last $2 \mathrm{~s}$, open circles) ripple stimuli for 16 electrodes. Electrode 16 represents the highest frequency channel.

spondees, two-syllable words with equal emphasis on each syllable, were recorded by a female talker. Two background noises were used: two-talker babble (one male and one female) and steady-state, speech-shaped noise. The female talker for the babble was different from the female talker for the spondees. The same background noise was used on every trial in order to eliminate variance that might arise from variability in the background stimulus. The onset of the spondees was $500 \mathrm{~ms}$ after the onset of the background noise. The two-talker babble and steady-state noise had duration of $2.0 \mathrm{~s}$. The level of the speech was held constant at $65 \mathrm{dBA}$, while the level of the noise was tracked using a one-down, one-up procedure and 2-dB steps. Feedback was not provided. The threshold for a single test run was estimated by averaging the signal-to-noise ratio for the final 10 of 14 reversals. The primary dependent variable was the mean SRT of the six test runs.

\section{Word recognition in quiet test}

Fifty consonant-nucleus-consonant (CNC) monosyllabic words (e.g., "Home, June, Pad, Sun") recorded by a male talker were presented, from an open set, in sound-field at 65 dBA (Peterson and Lehiste 1962). Each CNC word list was randomly chosen out of 10 lists for each subject. A total percent correct score was calculated as the percent of words correctly repeated. Feedback was not provided.

Results.

1. Spectral-ripple threshold

For the single-interval procedure, the mean threshold for the $14 \mathrm{CI}$ subjects was 6.16 ripples/octave with a $95 \%$ confidence interval of 1.70 ripples/octave. For the three-interval procedure, the mean threshold was 2.12 ripples/octave with a $95 \%$ confidence interval of 0.56 ripples/octave.

\section{Characteristics of psychometric function}

For the three-interval procedure, Won et al. (2007) showed a psychometric function in which there was a monotonic decrease as a function of ripple density with an upper asymptote in the ability to discriminate the standard and inverted ripple stimuli. The psychometric functions constructed from the single-interval procedure showed a similar 

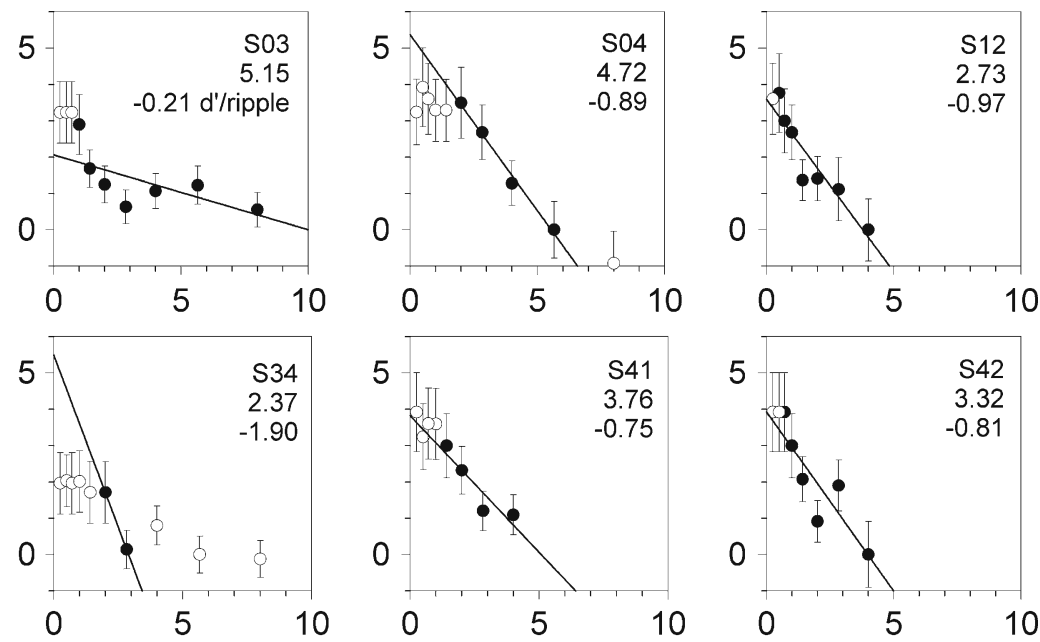

FIG. 3. Psychometric functions for single-interval spectral-ripple discrimination for $14 \mathrm{Cl}$ subjects. Each panel represents data for an individual subject. Data points used for linear regression fits are shown as filled circles. Linear fits are shown as solid lines. The second number shown in upper right corner in each panel shows threshold. The third number shown in upper right corner shows psychometric-function slope (in units of $d^{\prime} /$ ripple). Error bars show $80 \%$ confidence intervals. Open circles represent points other than those used to estimate the slope (e.g., upper and lower asymptotic points).
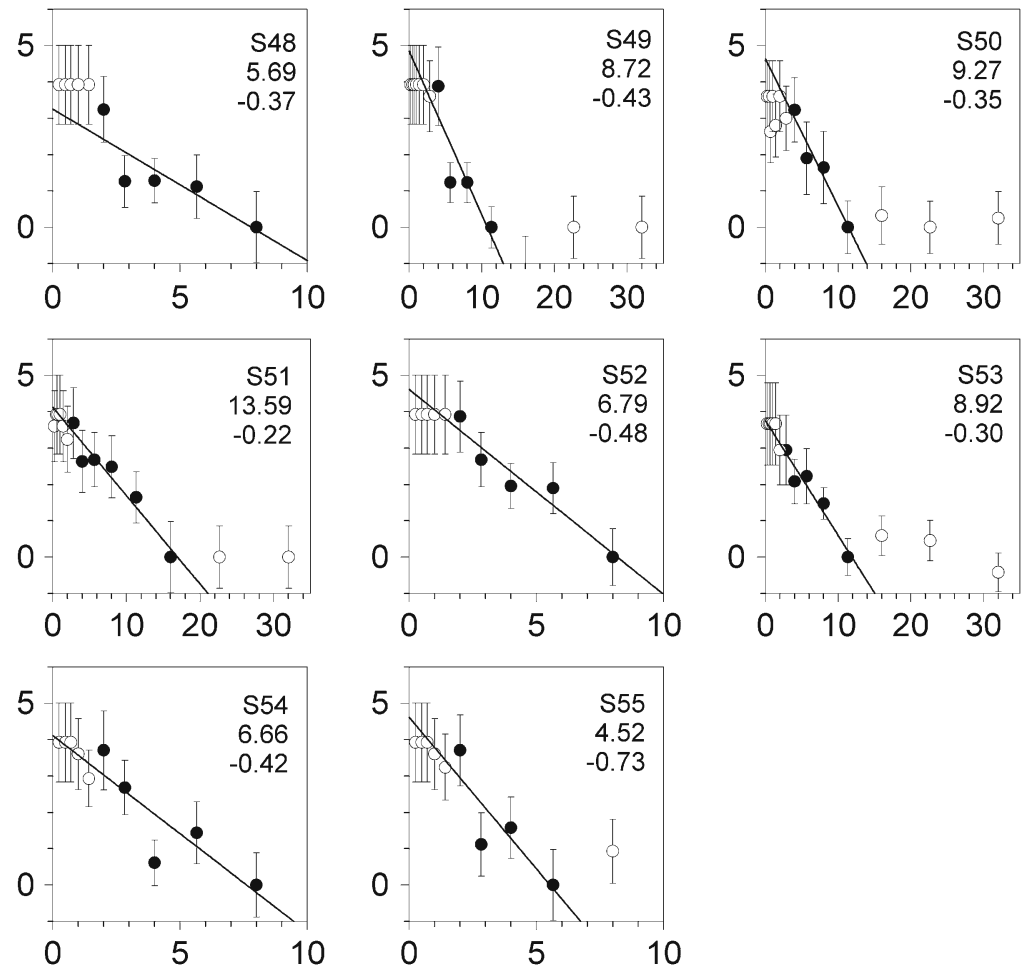

Ripples/octave

pattern. Figure 3 shows the psychometric functions obtained from the single-interval procedure method, plotting $d^{\prime}$ as a function of ripple density for each of the $14 \mathrm{CI}$ subjects. In the slope region, $d^{\prime}$ monotonically decreased as ripple density increased. Calculated slope values are indicated in each panel (e.g., $-0.89 d^{\prime}$ /ripple for S04) .

3. Comparing thresholds obtained from the singleand three-interval procedures

Figure 4 shows the threshold obtained from the single-interval procedure was correlated with the threshold obtained from the three-interval procedure for the 14 subjects. A highly significant correlation was found between the two methods $(r=0.84, p=0.0002)$.

4. Correlation between the single-interval spectralripple test and speech measures

Figure 5 shows the single-interval spectral-ripple thresholds significantly correlated with speech perception performance. Significant negative correlations were found between the single-interval spectralripple thresholds and SRTs in two-talker babble $(r=-0.64, p=0.01)$ and in steady-state noise $(r=-0.68$, $p=0.008)$. A significant correlation was also found between the single-interval spectral-ripple thresholds 


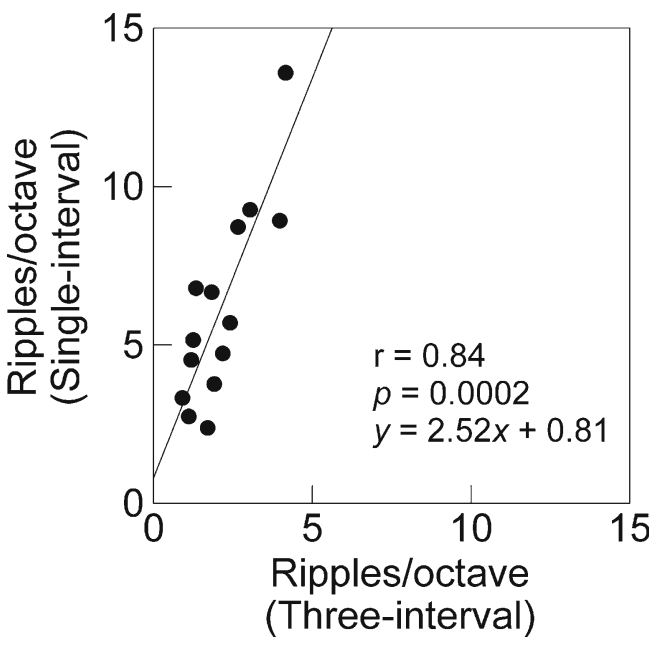

FIG. 4. Relationship between spectral-ripple thresholds determined using the three-interval procedure and those derived from the singleinterval procedure in $14 \mathrm{Cl}$ subjects. Linear regression is represented by the solid line.

and CNC word recognition scores in quiet ( $r=0.72, p=$ $0.004)$. These results were consistent with previously reported data from the three-interval procedure (Won et al. 2007; Henry et al. 2005). Table 2 shows correlations between $d^{\prime}$ values at each ripple density and speech perception scores.

Discussion. In experiment 1 , a significant correlation $(r=0.84)$ was found between the thresholds obtained using the two procedures. Most importantly, consistent with previous reports by Won et al. (2007), there were significant correlations between the single-interval spectral-ripple test and speech perception in the presence of two-talker babble $(r=-0.64, p=0.01)$, steady-state noise $(r=-0.68, p=0.0008)$, and with CNC word recognition scores $(r=0.72, p=0.004)$. With that said, caution needs to be taken when interpreting correlation results because they do not necessarily mean that the same hearing mechanisms were involved during the single-interval ripple test, threeinterval ripple test, and speech perception tasks. The integrity of the auditory systems of CI users having excellent three-interval spectral-ripple thresholds might be quite good and thus show overall good performance. Even so, our results suggest that the single-interval, yes/ no paradigm is a comparable method for evaluating spectral-ripple discrimination in CI users.

The mean spectral-ripple threshold was 6.16 ripples/octave for the single-interval test and 2.12 ripples/octave for the three-interval test. The higher thresholds obtained using the single-interval test compared to the three-interval test might, in part, be explained by procedural differences between the two methods. In the single-interval method, listeners were presented with either a single standard-standard ripple or standard-inverted ripple stimulus that was $4 \mathrm{~s}$ in duration. Thus, listeners could use the ripple phase inversion at the 2-s midpoint in the singleinterval procedure, thereby providing an immediate comparison between the two portions of the same stimulus (see Figs. 1 and 2). This immediate comparison might involve a sensory-trace comparison as described by Durlach and Braida (1969) as well as Kidd et al. (1988) during spectral shape discrimination. In contrast, the three-interval procedure included three stimuli whereby an inverted ripple stimulus was presented along with two other standard ripple stimuli. The three-interval procedure required that listeners select the interval that sounded different from the other two, a task that likely requires more demand on short-term memory and other cognitive abilities to make the comparison. We speculate that this additional cognitive load contributed to poorer ripple thresholds. In addition, the duration of the single-interval stimulus for each ripple phase is longer (2 s) than the interval duration of the 3-AFC stimuli (500 ms), so the subjects had a longer duration for comparison with the single-interval test. Another
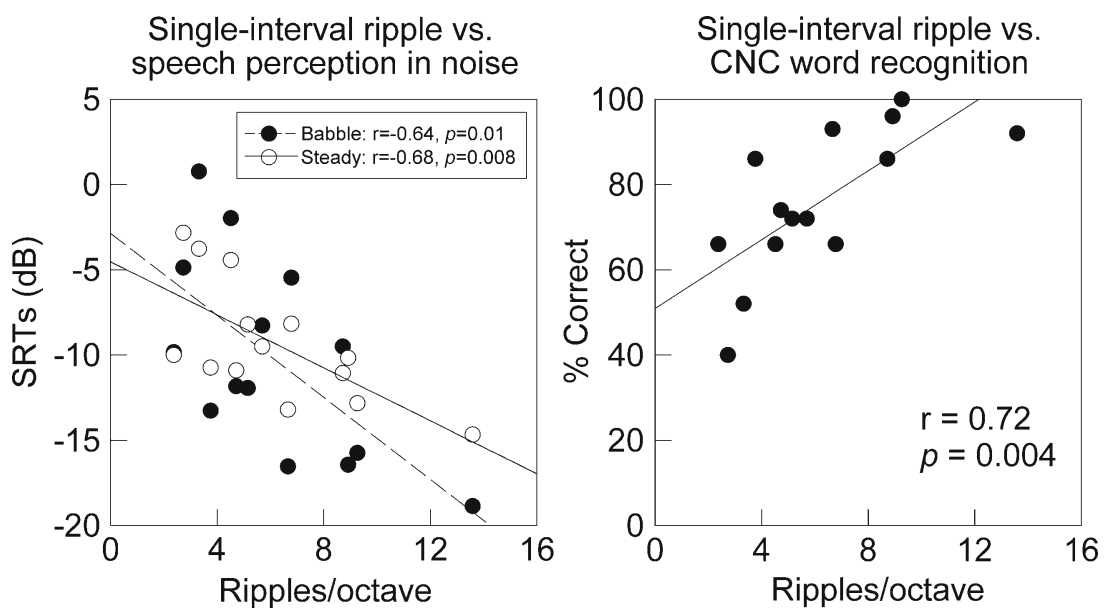

FIG. 5. Relationship between singleinterval spectral-ripple thresholds and speech reception thresholds in noise (left panel) and CNC scores (right panel). Linear regressions are represented by the dashed line for two-talker babble and the solid line for steady-state noise in the left panel. 
TABLE 2

Correlations of $d^{\prime \prime}$ values at each ripple density with speech perception tests and 3-AFC ripple test

\begin{tabular}{llllllrrrr}
\hline RIPPLE DENSITY & 0.25 & 0.5 & 0.707 & 1 & 1.414 & 2 & 2.828 & 4 & 5.657 \\
$\begin{array}{l}\text { Speech in babble } \\
\text { Speech in steady noise }\end{array}$ & & & & & & & & \\
CNC word & & & 0.57 & 0.54 & 0.61 & -0.54 & 0.59 & 0.63 & 0.68 \\
3-AFC ripple & & & & 0.55 & & 0.65 & 0.64 & 0.67 & 0.65 \\
\hline
\end{tabular}

Correlation values with $p<0.05$ are only shown. Nonsignificant correlations are not shown and left blank

difference is that, the single-interval procedure used the method of constant stimuli and estimated a threshold that corresponded to a $d^{\prime}=1$, whereas the three-interval procedure used a two-up, one-down adaptive procedure, converging on $70.7 \%$ correct point (i.e., $d^{\prime}=1.28$ ) on the psychometric function. Although the differences between the two procedures could contribute to the elevated spectral-ripple threshold in the single-interval procedure, it should be emphasized that the high, significant correlations between the results obtained with the two methods and the speech recognition scores suggest that the single-interval test can be used to evaluate spectral-ripple discrimination in CI listeners.

Table 2 shows correlations between $d^{\prime}$ values at each ripple density and speech perception performance and spectral-ripple thresholds obtained with 3AFC paradigm. Generally, $d^{\prime}$ at the slopes showed significant correlations with speech perception, whereas $d^{\prime}$ at the coarsest density did not. At lower ripple densities, listeners could have used intensity cue, but the $d^{\prime}$ values at lower ripple densities did not correlate with speech perception or with spectral-ripple thresholds obtained with the 3-AFC ripple test. On the contrary, the $d^{\prime}$ values at higher ripple densities, where spectral sensitivity is required for discrimination, were significantly correlated with speech perception and the 3-AFC spectral-ripple thresholds. These results further suggest that the single-interval ripple test evaluates spectral sensitivity which correlates with speech perception in CI users.

Experiment 2: the effect of number of channels on behavioral and physiological spectral-ripple threshold

In experiment 2, behavioral and physiological measures of spectral-ripple resolution were done with NH listeners. The single-interval procedure stimuli from experiment 1 were used for cochlear implant vocoder simulations. The effect of the number of vocoder processing channels on both behavioral and physiological spectral-ripple resolution was also investigated. The CI vocoder simu- lations serve as a model of CI users' performance. We chose to test normal-hearing subjects listening to CI vocoder simulations to avoid confounding artifacts when recording cortical responses from CI users (e.g., Friesen and Picton 2010). Because cortical P1-N1-P2 "change" responses can be recorded using the spectral-ripple stimuli processed with the vocoder simulation, it suggests that it is feasible to use the single-interval spectralripple stimuli to explore the behavioral-physiological relationship of spectral-ripple discrimination in CI users.

Subjects. Three NH listeners participated. Each had audiometric thresholds less than $15 \mathrm{~dB} \mathrm{HL}$ at all octave frequencies tested (from 0.25 to $8 \mathrm{kHz}$ ). The ages of the subjects were 27, 28, and 30 .

\section{Stimuli and procedures.}

1. Vocoder simulation of single-interval spectral-ripple discrimination

To assess the effect of the number of channels on the single-interval spectral-ripple test, vocoder simulations of the stimuli were presented to the NH listeners. The same stimuli used in experiment 1 were used in this experiment. The number of channels tested included 1, 4, 8, 16, and 24. For vocoder stimuli, ripple densities of $0.25,0.5,1,2,4$, and 8 ripples/octave were used. The input stimuli were divided into subband signals between 80 and $17,640 \mathrm{~Hz}$ by using a variable number of band-pass finite impulse response filters (e.g., Rubinstein and Turner 2003; Drennan et al. 2007; Won et al. 2008). For each number of channels condition, the center frequencies of the analysis filters are equally spaced along the human cochlear frequency map (Greenwood 1990). The envelope and the fine structure were extracted using a Hilbert transform. The Hilbert phases of the subband signals were then randomized, which creates a noise-band vocoder (Shannon et al. 1995). The randomized fine-structure of each sub-band was multiplied by the Hilbert envelope of each sub-band signal. Finally, the same band-pass filter was applied again to the sub-band output and all filtered sub-band outputs were summed together. The single-interval, yes/no paradigm spectral-ripple discrimination test was 
administered as previously described in experiment 1. The 1-, 4-, 8-, 16-, and 24-channel conditions were run in random order.

\section{Electrophysiological methods}

In the electrophysiological procedure, the same single-interval stimuli and vocoder simulation described above were used (ripple densities: 0.25, 1,4 , and 8 ripples/octave). To minimize recording time, the one-channel condition was not tested but the 4-, 8-, 16-, and 24-channel conditions were randomly presented. Each ripple density condition involved 200 presentations of standard-inverted ripple stimuli. During behavioral and electrophysiological testing, stimuli were presented at $65 \mathrm{dBA}$ in the free field in a sound-treated booth via a single loudspeaker, positioned $1 \mathrm{~m}$ from the subject. Stimulus onset asynchrony (the time from the onset of one stimulus to the onset of the next stimulus) was randomly jittered $(8,000 \mathrm{~ms} \pm 500 \mathrm{~ms})$ to reduce neural adaptation. Evoked potentials were recorded with a 64-channel electrode cap using a PC-based Neuroscan $^{\mathrm{TM}}$ system. Analog-to-digital sampling rate was $1,000 \mathrm{~Hz}$. The reference electrode was $\mathrm{Cz}$ (vertex) during recording and was re-referenced offline to an average reference. The recording window was $5,100 \mathrm{~ms}$ in duration, including a $100 \mathrm{~ms}$ pre-stimulus baseline. Online EEG filters were $0.05-100 \mathrm{~Hz}$. Data were low-pass filtered offline at $40 \mathrm{~Hz}$. Ocular artifact was monitored with electrodes at the lateral canthus of each eye and with electrodes inferior and superior to each eye. Sweeps including artifacts exceeding $\pm 70 \mu \mathrm{V}$ were not included in the average.

All recordings were obtained in a sound-treated booth while the subject sat in a comfortable chair attending to a muted, close-captioned movie of their choice. Each subject completed data collection over the course of two sessions on different days, lasting approximately $10 \mathrm{~h}$. There were 5-min breaks between each condition. The order of conditions was randomized. Evoked potentials were recorded on separate days following the behavioral test.

P1-N1-P2 responses, signaling stimulus onset, change, and stimulus offset were detected using visual analysis, as well as a statistical test applied to the phase coefficients of a wavelet transform (Ross et al. 1999, 2007). Individual accepted sweeps from the averagereferenced $\mathrm{Cz}$ electrode were submitted to a Morlet wavelet transform, resulting in amplitude and phase coefficients for each point in time and frequency. The Rayleigh test (Fisher 1993) was performed on the phase coefficients from the wavelet analysis. If the phase of a time-frequency point was uniformly distributed across the epochs, then the null hypothesis would be accepted, indicating that there was no response to the stimulus change in that particular condition.

\section{Results.}

1. Behavioral threshold as a function of the number of channels

The filled circles in Fig. 6 show the behavioral spectral-ripple thresholds as a function of the number of channels. A one-way repeated measure of ANOVA was performed to compare threshold with the number of channels, and as expected, there was a significant effect of the number of channels $\left(F_{4,9}=\right.$ 19.95, $p<0.001$ ). Post hoc tests (Bonferroni) showed a statistically significant difference between the 24 channel and all other channel conditions. This is consistent with a previous observation in NH listeners that the spectral-ripple discrimination ability increases as the number of channels increases (Henry and Turner 2003).

2. Physiological response as a function of the number of channels

Individual evoked cortical responses are shown in Fig. 7A (subject N1, left panel) and Fig. 7B (subject $\mathrm{N} 2$ and N3). Onset P1-N1-P2 responses were seen in all subjects in all conditions. P1-N1-P2 "change" responses, elicited by spectral-ripple phase inversions, were also seen in all subjects, and the presence or absence of the "change" response approximates behavioral thresholds (shown as a $\theta$ in Fig. 7A and B).

$P$ values resulting from the Rayleigh test are shown for one subject in the right panel of Fig. 7A. The Rayleigh test was used as a statistical means of detecting the presence of the "change" response; results from the Rayleigh test were consistent with behavioral thresholds. The presence of a physiological

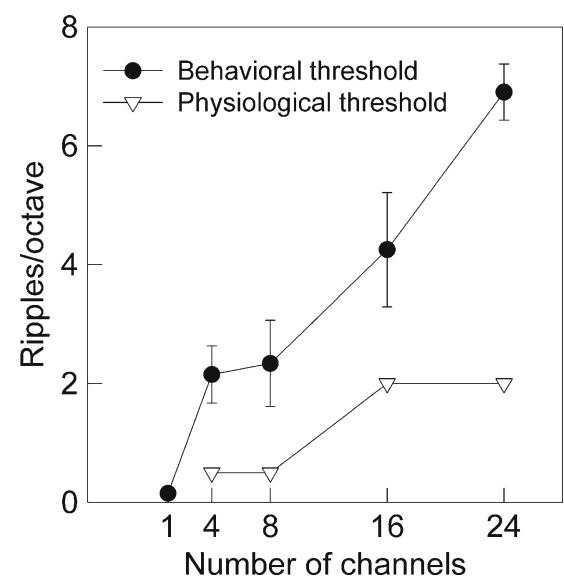

FIG. 6. Behavioral and physiological thresholds from experiment 2 as a function of number of vocoder processing channels. Error bars show standard error across subjects. 
"change" response was represented by low $p$ values in the 2.1-2.4 s range. When the "change" responses were present in time domain waveforms (left panel for each subject), the Rayleigh test had low $p$ values, less than 0.001, in the same latency region where the "change" response was observed (right panel for each subject). The presence of the "change" response, when analyzed visually using the time-domain waveform, as well as by the objective Rayleigh test, approximated behavioral thresholds. Similar results were obtained when electrodes over temporal lobes were examined.

The open triangles in Fig. 6 show the estimated physiological thresholds as a function of the number of channels. Physiological thresholds were estimated as the geometric mean between the highest ripple density at which the "change" response was elicited (confirmed by the Rayleigh test) and the next ripple density. The physiological thresholds were lower than the behavioral thresholds for all conditions, but the

\section{A \\ N01}

Time domain

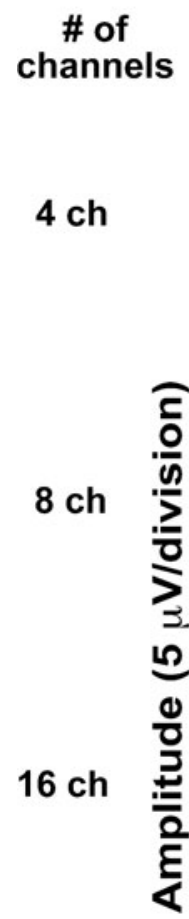

onset change offset
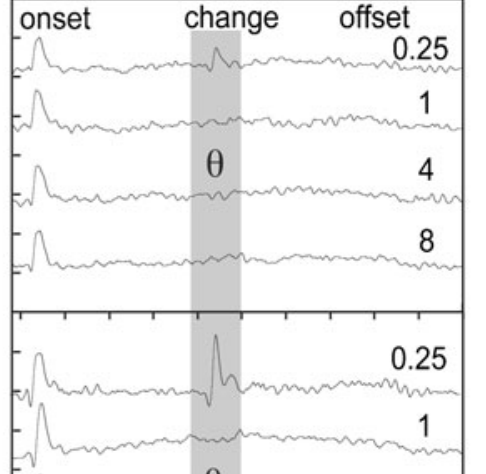

$24 \mathrm{ch}$

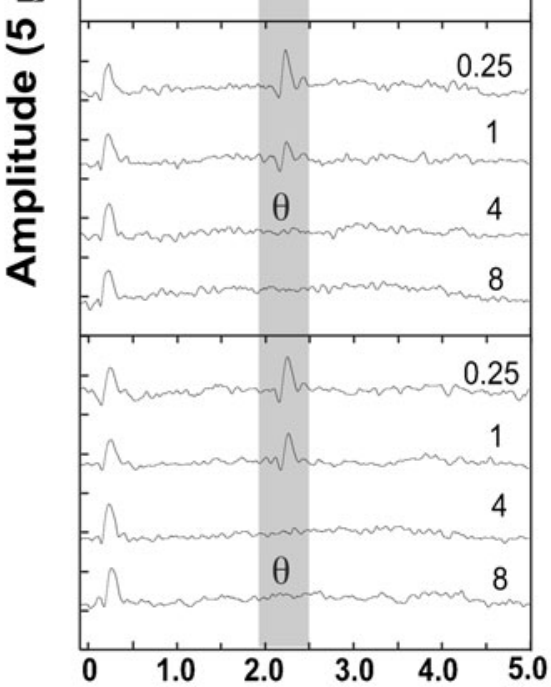

$\theta$

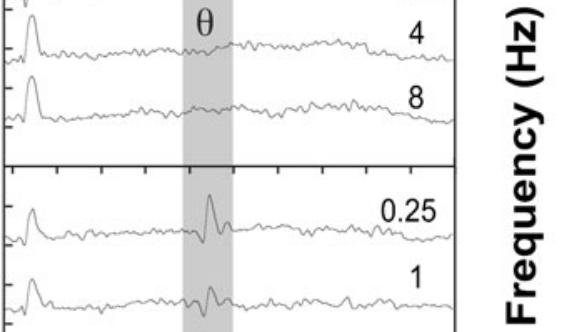

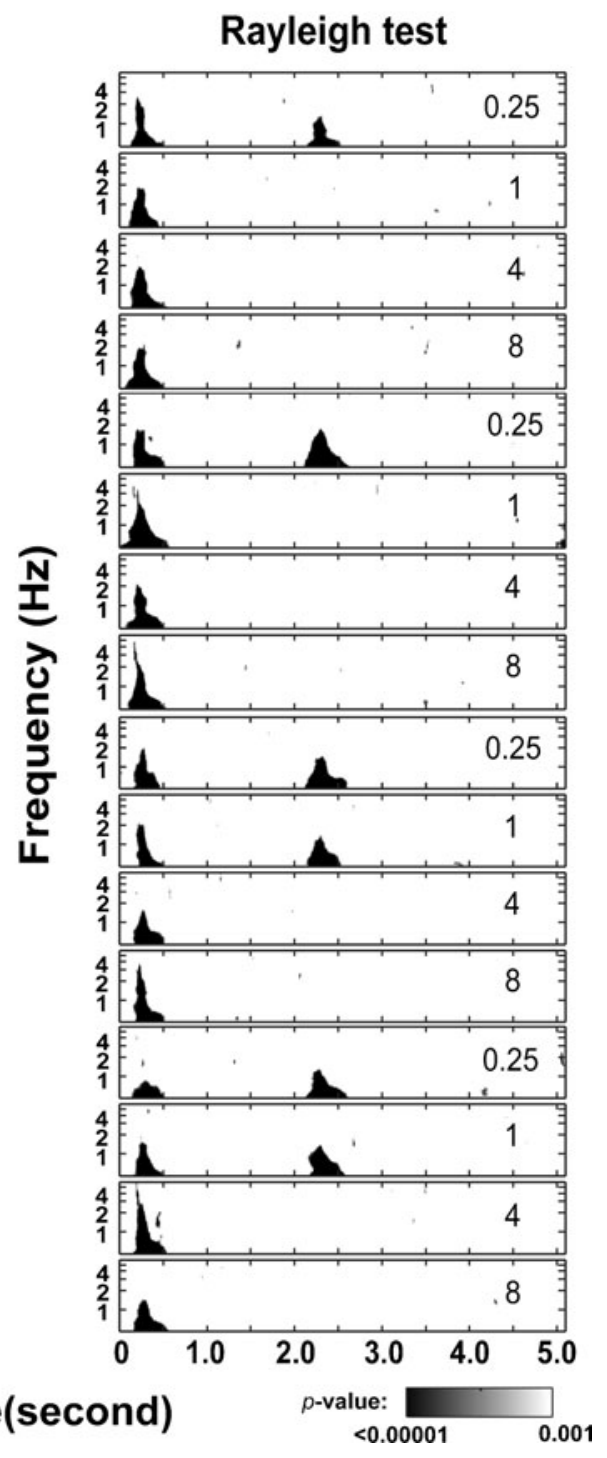

FIG. 7. Individual data from experiment 2. A shows physiologic data for subject N01. Left time-domain cortical auditory evoked potential waveforms are plotted from electrode $\mathrm{Cz}$. Each row represents a different vocoder channel condition (e.g., 24 channel, bottom row). Within each panel, responses to standard-inverted ripple stimuli are shown. The corresponding ripple density is displayed next to each waveform (e.g., 0.25 , top trace in each row). For comparison, the behavioral vocoder spectral-ripple discrimination thresholds are plotted as theta symbols.

Right for each cortical auditory evoked potential waveform, results of the Rayleigh test applied to coefficients of a time-frequency analysis of the cortical auditory evoked potential waveforms are plotted. $P$ values less than 0.001 from the Rayleigh test are indicated by the shaded areas. Statistically significant "onset" responses are present prior to $0.5 \mathrm{~s}$, while the "change" responses are represented by low $p$ values in the $2.1-2.4 \mathrm{~s}$ time range. B shows time-domain cortical auditory evoked potential waveforms for two subjects, N02 and N03. 


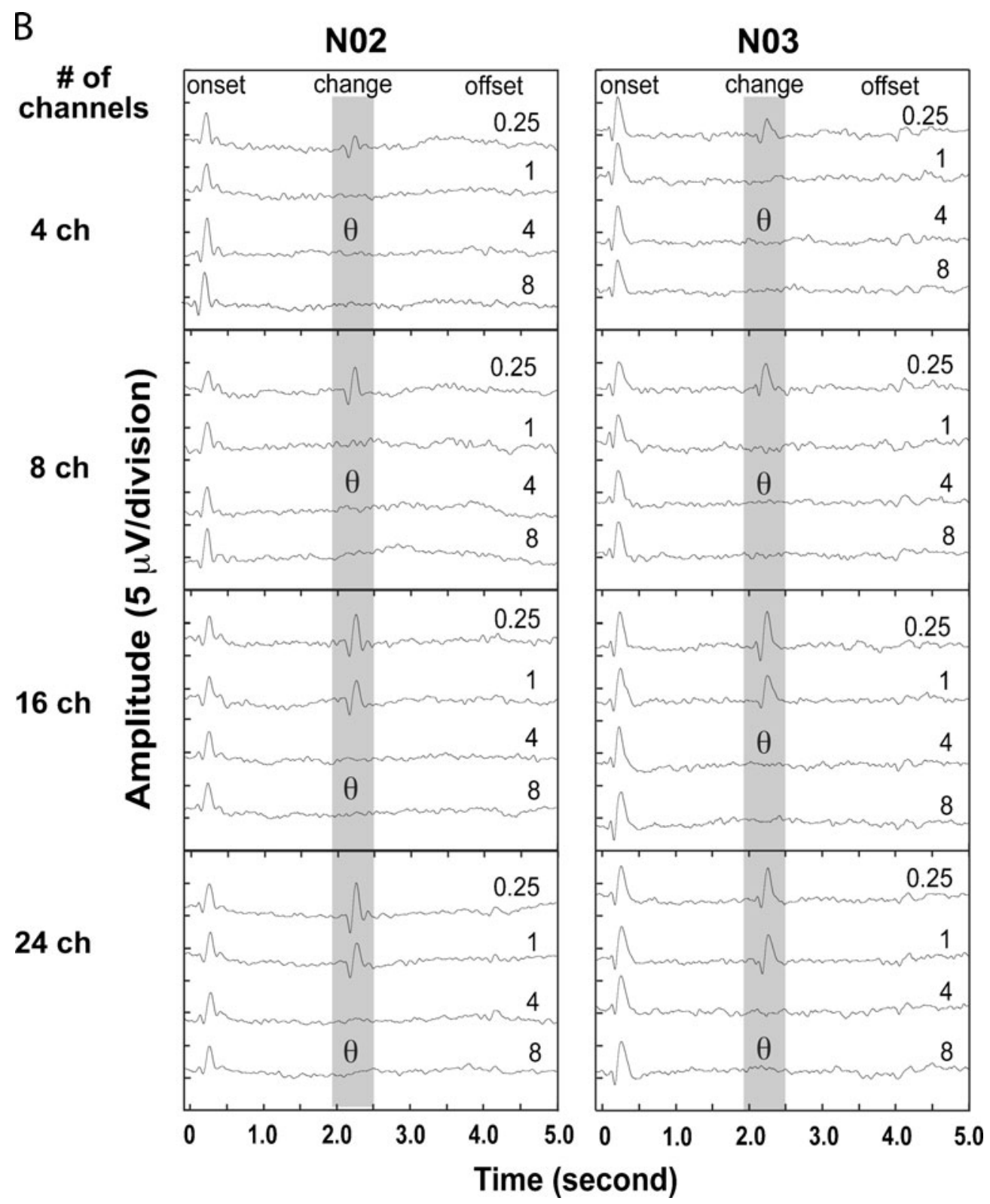

FIG. 7. (continued).

physiological thresholds also improved as a function of number of channels.

Discussion. In experiment 2, the vocoder behavioral data showed that $\mathrm{NH}$ listeners used the additional spectral information provided by higher numbers of channels to resolve more closely spaced spectral peaks to detect the change within a stimulus. Our observation is consistent with previous reports such as Henry and Turner (2003), which showed significant increases in performance on spectralripple discrimination up to 16 vocoder processing channels in NH listeners. Friesen et al. (2001, 2009) also showed that performance of $\mathrm{NH}$ listeners on vowel and consonant recognition increased up to 20 vocoder processing channels.

The physiological spectral-ripple data show that the presence of physiological "change" responses approximated the behavioral psychometric functions. As the number of vocoder channels increased, cortical "change" responses were affected, suggesting that the physiological sensitivity to spectral-ripple phase inversion increased with additional spectral information. These findings are in agreement with the behavioral discrimination data obtained from the same subjects, using the same stimuli. This is the first documentation that physiological "change" responses 
can be recorded in humans in response to the phase inversion of spectral-ripple stimuli. Further, our results show good agreement between physiological and behavioral thresholds showing a similar growth function as the number of channels increased (Fig. 6). In this respect, the physiology data show that it is feasible to further determine how close the brain-behavior relationship can be established using these methods.

What is less clear is the comparison between the CI subjects and $\mathrm{NH}$ vocoder simulations. The mean threshold of the CI subjects is comparable to that of the NH subjects when the number of processing channels ranged between 16 and 24. This result is not consistent with our initial prediction, as well as previous reports that CI listeners have only about eight functional channels (e.g., Shannon et al. 1995; Dorman and Loizou 1997, 1998; Henry and Turner 2003). The vocoder simulation in experiment 2 used the low and high cutoff frequencies of 80 and $17,640 \mathrm{~Hz}$, respectively. This frequency range is much wider than a typical CI processor (e.g., 250-8,700 Hz). This frequency range is also much wider than the $5,000 \mathrm{~Hz}$ bandwidth of the stimuli, thus several higher channels carried no information. If this discrepancy is considered, the mean threshold of CI subjects would correspond to the $\mathrm{NH}$ vocoder performance obtained with 11-17 simulated channels. It may be that, if the $\mathrm{NH}$ subjects were tested with a vocoder simulation covering 250-8,700 Hz, the mean threshold of the CI subjects would correspond to the NH vocoder simulation threshold at about eight channels. In addition, tone vocoders would also present better spectral contrast between standard and inverted ripple for the 3-AFC and the single-interval tests. These ideas were tested in experiment 3. Electrophysiology data were also collected using vocoder stimuli with these new filter settings, and utilized smaller steps in ripple density to better define physiological thresholds and the brain-behavior relationship.

\section{Experiment 3: the correlation between behavioral performance and change response amplitude}

In the behavioral measures for experiment 3 , noise and tone vocoder simulations were tested to determine if vocoder carrier affects spectral-ripple thresholds. Two channel conditions were tested including eight and 16 channels. The vocoder frequency range was 250 and 8,700 Hz. Electrophysiology data were also collected using smaller steps in ripple density to more precisely estimate electrophysiological thresholds so they could be compared to behavioral data from the same subjects.

Subjects. The same three NH listeners in experiment 2 participated. The subjects completed data collection over the course of multiple sessions on different days, lasting approximately $10 \mathrm{~h}$.

\section{Stimuli and procedures.}

1. Vocoder simulation and behavioral tests

A total of four vocoder simulations were created, including eight-channel noise, eight-channel tone, 16channel noise, and 16-channel tone vocoders. Ripple densities of $0.25,0.5,1,2,4$, and 8 ripples/octave were used. These ripple densities were also used in the behavioral single-interval test in experiment 2 and were wide enough to estimate the behavioral thresholds with eight- and 16-channel conditions. The noise vocoders used the same signal processing as experiment 2 except for different low cutoff $(250 \mathrm{~Hz})$ and high cutoff $(8,700 \mathrm{~Hz})$ frequencies. The tone vocoders also used the same signal processing as the noise vocoders except that the Hilbert envelope was modulated with a tone carrier at the center frequency of the band. Subjects were tested with a total of eight testing conditions in random order (i.e., four vocoder simulations for both 3-AFC and single-interval tests).

\section{Electrophysiology methods}

The same recording and analysis procedures were used as in experiment 2. For the electrophysiological data collection, only the eight-channel noise vocoder simulation was used. The electrophysiological data with the eight-channel condition in experiment 2 (see Fig. 4) were collected with $0.25,1,4$, and 8 ripples/ octave stimuli; "change" responses were not seen at densities greater than 0.25 ripples/octave for all subjects, suggesting that the physiological threshold was likely between a ripple density of 0.25 and 1 ripple/octave. In this experiment, each subject was tested at six ripple densities that were logarithmically equally spaced between 0.25 and 2 ripples/octave. These ripple densities were chosen to more closely approximate the physiological threshold by including ripple densities above and below the behavioral threshold.

\section{Results.}

\section{Behavioral thresholds}

Figure 8 shows the mean thresholds of the three $\mathrm{NH}$ subjects from the 3-AFC test (left panel) and the singleinterval test (right panel). For comparison, the mean spectral-ripple thresholds for the 14 CI subjects are indicated as filled triangles. A $2 \times 2 \times 2$ repeated measures ANOVA was performed on the thresholds (two types of tests, two carriers, and two channel numbers). It showed a significant effect of channel numbers $\left(F_{1,2}=\right.$ $55.8, p=0.017$ ), but the type of test (3-AFC or one interval) and carrier did not have any effect. Unlike the CI subjects, the NH subjects showed very similar performance on the 3-AFC and single-interval tests. When the NH vocoder thresholds were compared to the mean threshold of the 14 CI subjects, the eight-channel 


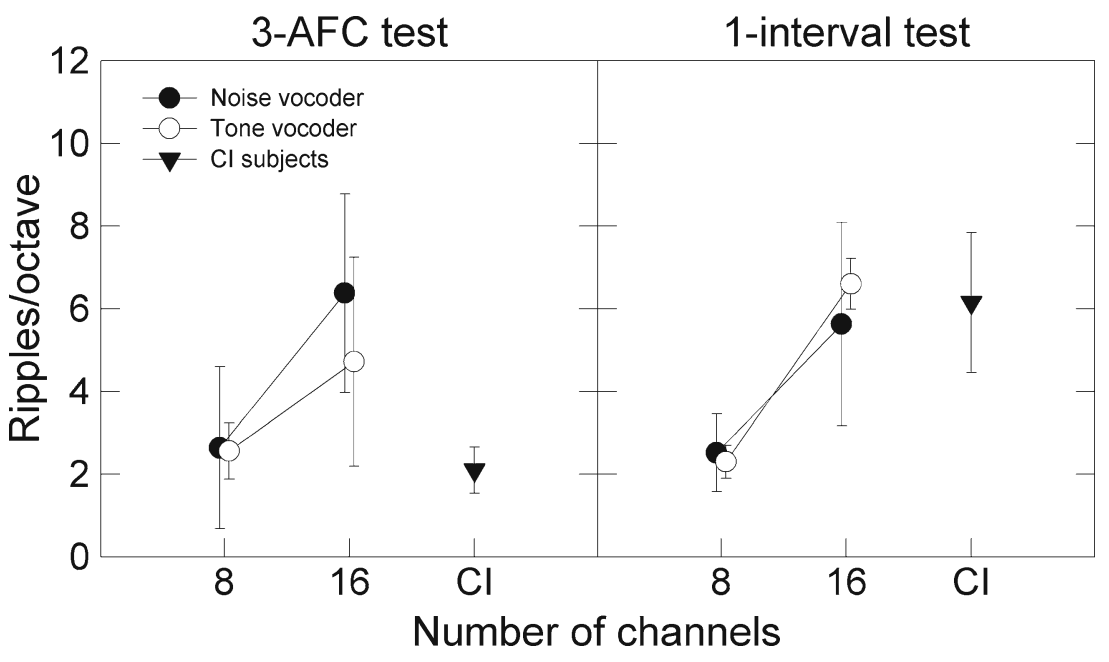

FIG. 8. Average spectral-ripple thresholds for four different vocoder conditions in $\mathrm{NH}$ listeners (left 3-AFC test, right one-interval test). For comparison, the mean spectral-ripple threshold for the $14 \mathrm{Cl}$ subjects is indicated as filled triangles. Error bars represent 95\% confidence intervals. Data points are slightly horizontally displaced for clarity. vocoder thresholds were comparable to the mean of the CI subjects for the 3-AFC test.

\section{Electrophysiological thresholds}

Figure 9 shows individual waveforms of cortical responses. The Rayleigh test was also performed to determine if the "change" responses were present in the waveforms. The Rayleigh test showed that there were "change" responses up to 1.0, 0.7079, and 1.4125 ripples/octave for N01, N02, and N03, respectively. Correspondingly, physiological thresholds, defined as the geometric mean between the highest ripple density at which the "change" response was elicited and the next ripple density, were 1.188, 0.841, and 1.681 ripples/octave for N01, N02, and N03, respectively, whereas the behavioral thresholds with the same vocoder were 3.0, 2.83, and 1.74 ripples/ octave for N01, N02, and N03, respectively. Physiological thresholds were slightly lower than behavioral thresholds for all subjects.

3. Correlation between behavioral and physiological data

To better determine the relationship between behavioral discrimination and physiological responses, the peak-to-peak amplitudes of N1-P2 "change" responses for all ripple densities were obtained. Response amplitudes were measured only for those conditions where the Rayleigh test identified a "change" response as being present; amplitudes of zero were recorded for conditions where the Rayleigh test indicated an absent "change" response. For each individual subject, N1-P2 "change" response amplitudes were normalized by the subject's largest "change" response amplitude. Amplitudes and $d^{\prime}$ declined as ripple density increased. Figure 10 illus-
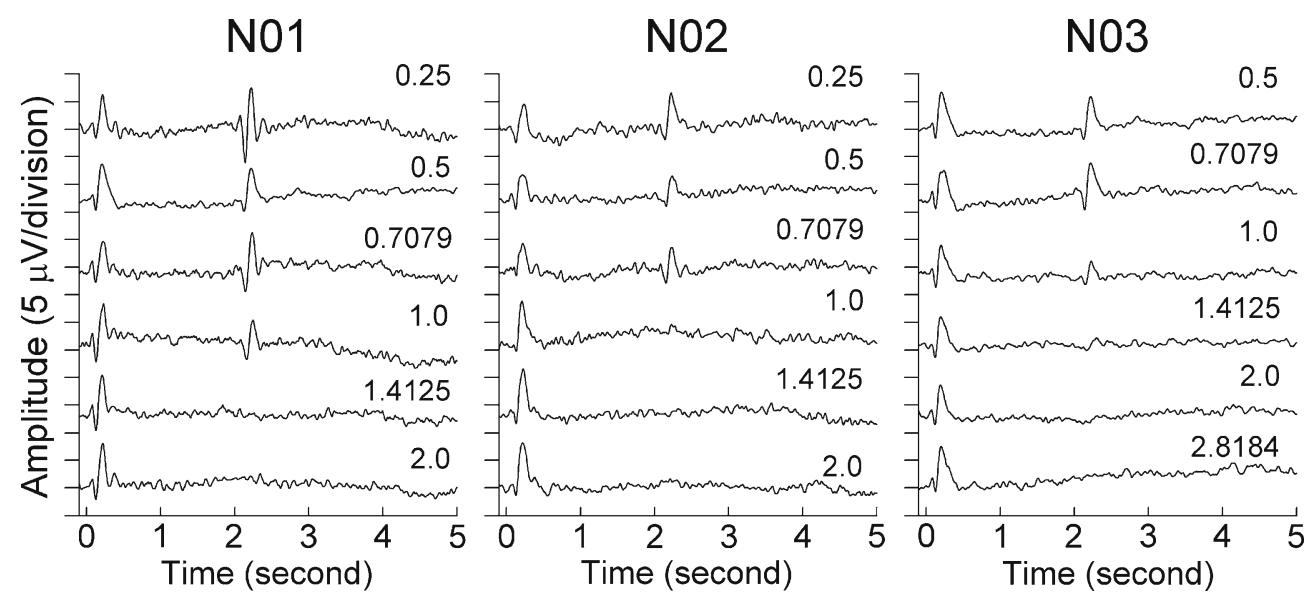

FIG. 9. Time-domain cortical auditory evoked potential waveforms are shown. Each column shows individual normal-hearing subject's data at different ripple densities (e.g., 0.25 ripples/octave). Stimuli were processed with the eight-channel noise vocoder simulation. The corresponding ripple density is displayed next to each waveform. Amplitude of "change" response decreases as ripple density increases. 


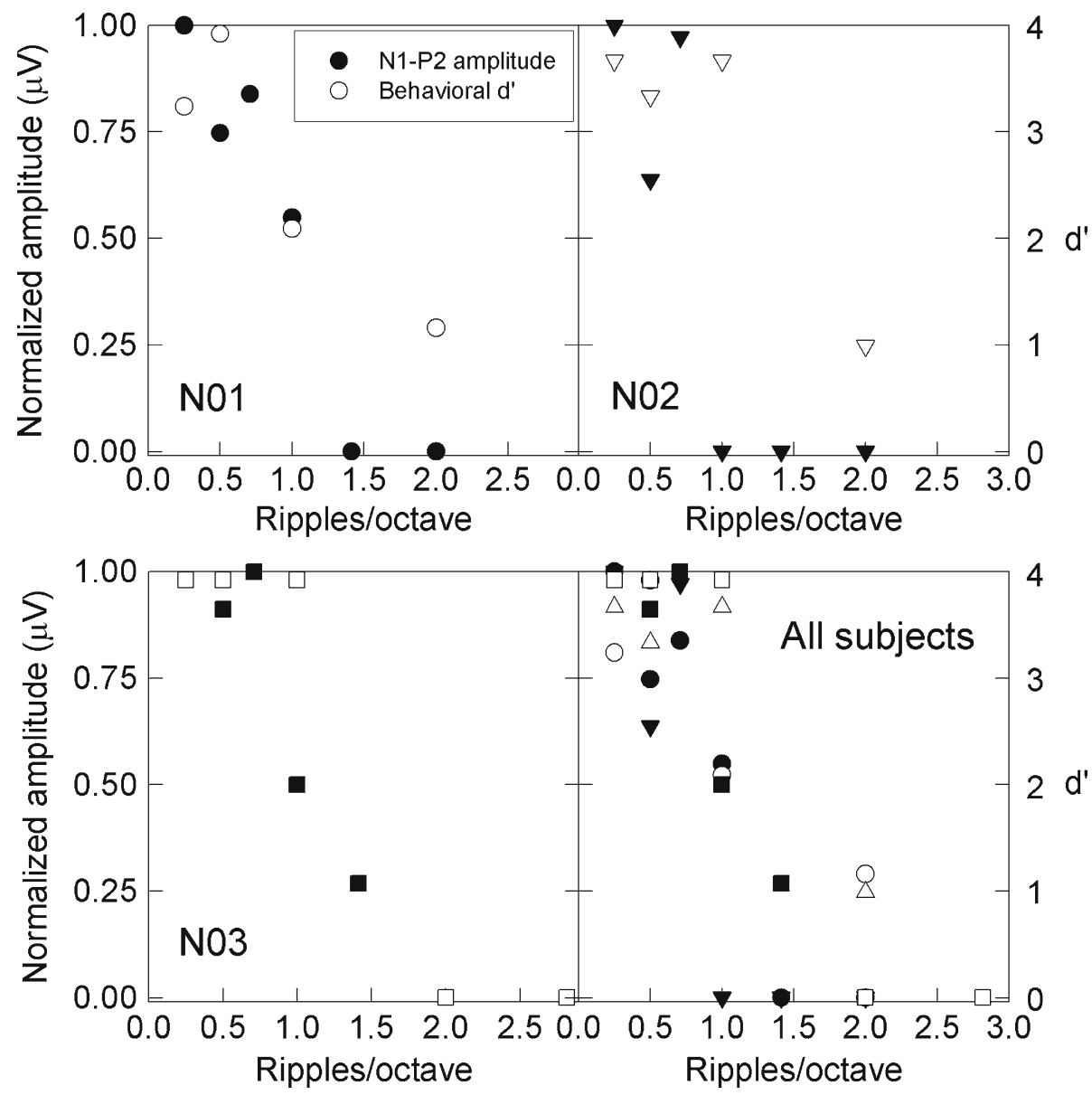

FIG. 10. Relationships between behavioral and physiological measures are illustrated in scatterplots of normalized amplitude of N1-P2 "change" responses (filled symbols plotted in $\mu \mathrm{V}$ on the left $y$ axis) and behavioral discrimination per-

$d^{\prime}$ formance (open symbols plotted in $d^{\prime}$ on the right $y$-axis) as a function of ripple density. Data for individual subject N01 (circles), N02 (triangles), and N03 (squares) are represented by different symbols.

trates the within-subject brain-behavior relationship and shows normalized N1-P2 response amplitudes and $d^{\prime}$ from the behavioral procedure. At low ripple densities, where $d^{\prime}$ was higher (i.e., better behavioral detection), N1-P2 "change" response amplitudes were also higher (i.e., better physiological detection).

Figure 11 illustrates the across-subjects brainbehavior relationship and shows the correlations between the normalized N1-P2 "change" response amplitudes and the $d^{\prime}$ values for all subjects at ripple densities in which both behavior and physiology were tested. There were strong correlations $(r \geq 0.86)$ observed for two subjects (N01 and N03). When all data points for the three subjects were pooled together to determine the correlation, about $54 \%$ of the variance $(r=0.73, p=0.007)$ in behavioral performance was accounted for by amplitudes of the N1-P2 "change" responses.

Discussion. When the new filter setting was used $(250-8,700 \mathrm{~Hz})$ to simulate the 3-AFC test, the mean threshold of CI subjects corresponded to the $\mathrm{NH}$, simulated eight-channel thresholds obtained with both noise and tone carrier vocoders. This observation suggests that the vocoder simulation signal processing is adequate and these data are consistent with previous reports of 8 functional channels in CI listeners (e.g., Shannon et al. 1995; Dorman and Loizou 1997, 1998; Henry and Turner 2003). For the single-interval test, the 16-channel vocoder threshold corresponded to the

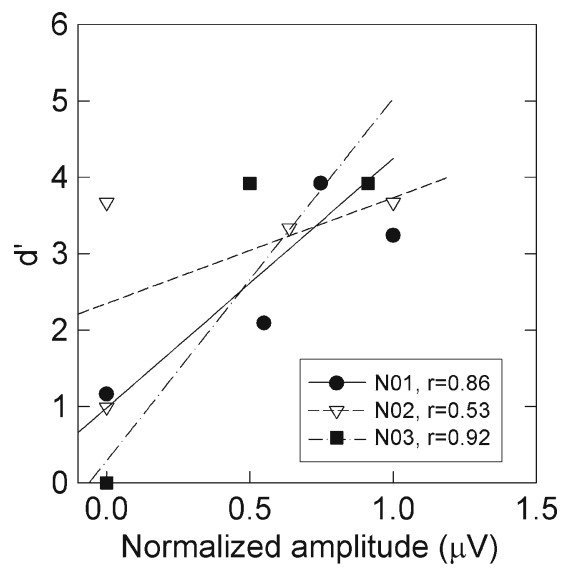

FIG. 11. The brain-behavior relationship is represented in scatterplots of the normalized amplitude of N1-P2 "change" responses and behavioral $d^{\prime}$ data. Data for individual subject N01 (circles), N02 (triangles), and N03 (squares) are represented by different symbols. Significant correlations were found for each subject; linear fits between the two measures are shown as lines for each subject. 
mean of the CI subjects. This result is not consistent with our prediction that the narrow filter range should result in the eight-channel single-interval vocoder threshold would correspond to the mean threshold of CI subjects. The NH vocoder simulation data in Fig. 8 showed no effect of test type (3-AFC vs. single-interval), whereas the CI subjects in experiment 1 showed significantly better threshold with the single-interval test.

It appears that in the single-interval test, some cues were only available to $\mathrm{CI}$ listeners and those cues were not preserved in the vocoder outputs. It might be that the output differences are attributed to the differences between the actual CI sound processing and the vocoder signal processing. To evaluate the output differences, the outputs of the actual CI sound processing and the noise and tone vocoder processing were compared. Fidelity 120 strategy was chosen for this analysis. The absolute value of the difference between output levels for standard and inverted ripple stimuli were estimated for individual channels. These values were summed across channels and the resulting value was used as an index to compare Fidelity 120 and the vocoder outputs. This analysis shows that the vocoder processing resulted in a much smaller index than Fidelity120, especially when the eight-channel vocoder was used. The vocoder simulations in this study did not implement pre-emphasis filters or compression functions. When they were added, a much larger index was observed. Other differences include frequency allocation for each channel, envelope detection method, input dynamic range, and automatic gain control. In addition, five of the 14 CI subjects used Fidelity120 as their clinical sound encoding strategy. Drennan et al. (2010) demonstrated that CI listeners could achieve significantly better spectral-ripple discrimination with Fidelity120 than with HiResolution, because current steering implemented in Fidelity120 could provide improved spectral detail of the sound. This pattern is also observed in the present study. The five users of Fidelity120 showed 7.4 ripples/octave, whereas the other nine subjects (i.e., non-Fidelity120 users) showed 5.5 ripples/octave for the one-interval test. Note that the vocoder simulation in the present study did not implement current steering. It seems that the vocoder simulated single-interval test was more affected by these differences. A future direction will be to define optimal recording parameters in case the ability to detect changes in ongoing acoustic vs. electric stimuli is differentially affected by neural adaptation (e.g., Wojtczak et al. 2003). Neural adaptation might help CI subjects detect changes at the 2-s point of the standard-inverted stimuli, whereas this effect might not be large enough for $\mathrm{NH}$ subjects and would be absent in the 3-AFC test. Nevertheless, the strong correlations of the single-interval spectral- ripple test in CI listeners with the 3-AFC spectralripple test and speech perception tests do not detract from the single-interval procedure as a potentially valuable measure of spectral-ripple discrimination.

The main finding from experiment 3 was that amplitudes of the "change" responses were correlated with behavioral performance. Electrophysiology data have also shown that the amplitudes of P1-N1-P2 "change" responses elicited by spectral-ripple phase inversion are related to the ripple density. That is, as ripple density increased, smaller frequency differences resulting from spectral-ripple phase inversion in the ongoing stimuli evoked lower amplitude "change" responses. The presence of "change" responses in the present data reflects the physiological detection of the acoustic cue in the stimuli, namely the physiological detection of spectralripple phase inversion at the level of the auditory cortex. The acoustic cue could also include a temporal component which disrupts an ongoing signal. Nevertheless, significant correlations between the behavioral and physiological data suggest that the neural encoding of spectral features in the acoustic sounds and its processing at the cortical level is an important factor in determining behavioral spectral-ripple discrimination. Although these correlations were obtained across conditions for individual subjects, a strong correlation was also found across subjects ( $r=0.73, p=0.007)$ when all subjects' data points were included for the correlation analysis.

When physiological thresholds were defined as the geometric mean between the highest ripple density at which the "change" response was elicited and the next ripple density; physiological thresholds were slightly lower than behavioral thresholds for all subjects. Different testing paradigms used in the behavioral and physiological conditions may have contributed to differences between the perceptual and physiological thresholds. For example, a passive listening paradigm was employed to record the physiology data, where cognitive processing and demands were different than during the behavioral listening task. To be of use clinically, it will be important to determine if the physiological detection of spectral-ripple change falls within a clinically acceptable range of perceptual thresholds, just as there is a clinically defined acceptable brain-behavior relationship when using auditory brainstem thresholds to estimate hearing sensitivity. Future directions will aim to optimize recording parameters, so clinically relevant relationships between physiological and perceptual "change" thresholds can be established using spectral-ripple stimuli.

\section{GENERAL DISCUSSION}

In the present study, a single-interval, yes/no paradigm spectral-ripple discrimination test was developed. When adult CI users were tested in 
experiment 1 , a highly significant correlation was found between the spectral-ripple threshold obtained using the proposed single-interval method and the threshold obtained using a previously established three-interval, three-AFC method (Won et al. 2007). The single-interval spectral-ripple threshold was also significantly correlated with speech recognition in quiet and noise. In experiment 2, the behavioral vocoder experiment showed that, as the number of channels increased, the $\mathrm{NH}$ listeners were able to detect and discriminate a higher density of spectralripple stimuli. The physiological test showed that the single-interval spectral-ripple stimuli can evoke the "change" response, signaling the physiological detection of spectral-ripple phase inversion, and the presence of this "change" response increased as a function of the number of vocoder processing channels. The presence of the change response qualitatively agreed with behavioral performance. In experiment 3, where smaller step sizes in ripple density were used, physiological thresholds were more accurately estimated. The amplitudes of the "change" responses significantly correlated with behavioral performance. Taken together, these findings confirm that the single-interval spectral-ripple discrimination is a feasible approach for studying spectral-ripple resolution in the human auditory system.

Several studies have compared different types of psychophysical procedures and threshold estimation methods such as single-, two-, or three-AFC procedures using either the adaptive staircase rule, maximum-likelihood stepping rule, or the method of constant stimuli (e.g., Shelton et al. 1982; Shelton and Scarrow 1984; Saberi and Green 1997; Leek 2001; Buss et al. 2001; Marvit et al. 2003). In general, different efficiency and reliability were found according to the type of hearing task. Likewise, it appears that both single- and three-interval spectral-ripple tests in the current study have their own distinct advantages.

From the perspective of testing time, the threeinterval procedure is preferred because it requires significantly shorter time to obtain a threshold than the single-interval procedure. However, the singleinterval procedure is more appropriate for naïve listeners (e.g., young children) who are less able to execute a task that requires comparing two or more separate sounds (Green 1995). Single-interval procedures are readily adaptable for testing preverbal children using observer-based psychophysical procedures, which have been used to investigate several hearing abilities in NH infants (e.g., Olsho et al. 1987; Bargones et al. 1995; Leibold and Werner 2006) as well as in infant CI users (Dasika et al. 2009; GriecoCalub et al. 2008).

The current study found a significant correlation of the single-interval spectral-ripple test with speech recognition in quiet as well as in noise, which suggests the intriguing possibility that if the spectral-ripple threshold can be obtained from prelingual CI users, their speech perception capabilities could be predicted. This clinical information could lead to improved habilitation/rehabilitation and educational interventions, as well as outcomes. In principle, such measures could also allow testing and optimization of sound processing strategies in children whose auditory nervous systems are still maximally plastic.

In addition, the spectral change stimuli used in the single-interval approach have also been demonstrated to be appropriate to conduct electrophysiological studies to explore spectral information processing in the auditory cortex. If cortical auditory evoked potentials can be measured in response to spectral-ripple phase inversion in the stimulus in CI listeners, it may be possible to evaluate various mapping strategies and possibly determine which strategy leads to the best spectral resolution. Once optimal stimulus presentation rates and recording montages for maturing auditory systems are defined, such approaches may facilitate the tracking of longitudinal and developmental changes involving spectral processing in CI users following implantation. All of these possibilities have motivated the present study, and the feasibility data obtained in this study justify expanding this area of research to include the single-interval approach when studying auditory spectral processing in $\mathrm{NH}, \mathrm{HI}$, and $\mathrm{CI}$ listeners.

\section{ACKNOWLEDGMENTS}

We greatly appreciate the dedicated efforts of our subjects. This study was supported by NIH-NIDCD grants R01DC007525, P30-DC04661, R01-DC007705, T32-DC000033, F31-DC009755，F31-DC010553，F32-DC008238， L30DC008490, and Korea Research Foundation grant KRF2006-612-D00106, and an educational fellowship from Advanced Bionics Corporation. Thanks to Bernhard Ross for his programming assistance with the Rayleigh test as well as his consultation.

\section{REFERENCES}

Abbas PJ, Brown CJ (2000) Electrophysiology and device telemetry. In: Waltzman SB, Cohen NL (eds) Cochlear implants. Thieme

Bargones JY, Werner LA, Marean GC (1995) Infant psychometric functions for detection: mechanisms of immature sensitivity. J Acoust Soc Am 98:99-111

Brown CJ, Hughes ML, Luk B, Abbas PJ et al (2000) The relationship between EAP and EABR thresholds and levels used to program the nucleus 24 speech processor: data from adults. Ear Hear 21:151-163

Buss S, Schorer E, Florentine M, Zwicker E (1986) Decision rules in detection of simple and complex tones. J Acoust Soc Am 80:1646-1657 
Buss E, Hall JW, Grose JH, Dev MB (2001) A comparison of threshold estimation methods in children 6-11 years of age. J Acoust Soc Am 109:727-731

Byrne D, Dillon H, Tran K, Arlinger S et al (1994) An international comparison of long-term average speech spectra. J Acoust Soc Am 96:2108-2120

Dasika VK, Werner LA, Norton SJ, Nie K et al (2009) Measuring sound detection and reaction time in infant and toddler cochlear implant recipients using an observer-based procedure: a first report. Ear Hear 30:250-261

Dorman MF, Loizou PC (1997) Speech intelligibility as a function of the number of channels of stimulation for normal-hearing listeners and patients with cochlear implants. Am J Otol 18: S113-S114

Dorman MF, Loizou PC (1998) The identification of consonants and vowels by cochlear implant patients using a 6-channel continuous interleaved sampling processor and by normal-hearing subjects using simulations of processors with two to nine channels. Ear Hear 19:162-166

Drennan WR, Won JH, Dasika VK, Rubinstein JT (2007) Effects of temporal fine structure on the lateralization of speech and on speech understanding in noise. J Assoc Res Otolaryngol 8:373383

Drennan WR, Won JH, Nie K, Jameyson EM, Rubinstein JT (2010) Sensitivity of psychophysical measures to signal processor modifications in cochlear implant users. Hear Res 262:1-8

Durlach NI, Braida LD (1969) Intensity perception. I. Preliminary theory of intensity resolution. J Acoust Soc Am 46:372-383

Eisenberg LS, Martinez AS, Boothroyd A (2007) Assessing auditory capabilities in young children. Int J Pediatr Otorhinolaryngol 71:1339-1350

Faulkner KF, Tremblay KL, Rubinstein JT, Werner LA, Nie K (2010) Auditory training in adult cochlear implant listeners using spectrally-rippled noise stimuli in an adaptive, single-interval paradigm. Presented at 33rd annual meeting of the association research in otolaryngology. Abstract \#363

FisHer NI (1993) Statistical analysis of circular data. Cambridge University Press, Cambridge

Friesen LM, Picton TW (2010) A method for removing cochlear implant artifact. Hear Res 259:95-106

Friesen LM, Tremblay KL (2006) Acoustic change complexes recorded in adult cochlear implant listeners. Ear Hear 27:678685

Friesen LM, Shannon RV, Baskent D, Wang X (2001) Speech recognition in noise as a function of the number of spectral channels: comparison of acoustic hearing and cochlear implants. J Acoust Soc Am 110:1150-1163

Friesen LM, Tremblay KL, Rohila N et al (2009) Evoked cortical activity and speech recognition as a function of the number of simulated cochlear implant channels. Clin Neurophysiol 120 (4):776-782

Gourevitch V, Galanter E (1967) A significance test for one parameter isosensitivity functions. Psychometrika 32:25-33

GREEN DM (1995) Maximum-likelihood procedures and the inattentive observer. J Acoust Soc Am 97:3749-3760

GreENWOOD DD (1990) A cochlear frequency-position function for several species-29 years later. J Acoust Soc Am 87:2592-2605

Grieco-Calub TM, Litovsky RY, Werner LA (2008) Using the observer-based psychophysical procedure to assess localization acuity in toddlers who use bilateral cochlear implants. Otol Neurotol 29:235-239

HARRIS RW (1991) Speech audiometry materials compact disk. Brigham Young University, Provo

Henry BA, Turner CW (2003) The resolution of complex spectral patterns by cochlear implant and normal-hearing listeners. J Acoust Soc Am 113:2861-2873
Henry BA, McKay CM, McDermott HJ, Clark GM (2000) The relationship between speech perception and electrode discrimination in cochlear implantees. J Acoust Soc Am 108:1269-1280

Henry BA, Turner CW, Behrens A (2005) Spectral peak resolution and speech recognition in quiet: normal hearing, hearing impaired, and cochlear implant listeners. J Acoust Soc Am 118:1111-1121

JERGER J, Jerger S (1970) Evoked response to intensity and frequency change. Arch Otolaryngol 91:433-436

Kidd G, Mason CR, Hanna TE (1988) Evidence for sensory-trace comparisons in spectral shape discrimination. J Acoust Soc Am 84:144-149

LEEK MR (2001) Adaptive procedures in psychophysical research. Percept Psychophys 63:1279-1292

LeIBOLd L, WERNER L (2006) Effect of masker-frequency variability on the detection performance of infants and adults. J Acoust Soc Am 119:3960-3970

LevitT H (1971) Transformed up-down methods in psychoacoustics. J Acoust Soc Am 49(Suppl 2):467-477

Litvak LM, Spahr AJ, SAoji AA, Fridman GY (2007) Relationship between perception of spectral ripple and speech recognition in cochlear implant and vocoder listeners. J Acoust Soc Am 122:982-991

MacMillan NA, Creelman CD (2005) Detection theory: a user's guide. Erlbaum, Hillsdale

Martin BA, Tremblay KL, Korczak P (2008) Speech evoked potentials: from the laboratory to the clinic. Ear Hear 29:285-313

Marvit P, Florentine M, Buus S (2003) A comparison of psychophysical procedures for level-discrimination thresholds. J Acoust Soc Am 113:3348-3361

McCandless GA, Rose DE (1970) Evoked cortical responses to stimulus change. J Speech Hear Res 13:624-634

Olsho LW, Косн EG, Halpin CF (1987) Level and age effects in infant frequency discrimination. J Acoust Soc Am 82:454-464

Peterson GE, Lehiste I (1962) Revised CNC lists for auditory tests. J Speech Hear Disord 27:62-70

Ponton CW, Don M, Eggermont JJ, Waring MD et al (1996) Auditory system plasticity in children after long periods of complete deafness. NeuroReport 8:61-65

Ross B, Lutkenhoner B, Pantev C, Hoke M (1999) Frequencyspecific threshold determination with the ceragram method: basic principle and retrospective evaluation of data. Audiol Neurootol 4:12-27

Ross B, Tremblay KL, Picton TW (2007) Physiological detection of interaural phase differences. J Acoust Soc Am 121:10171027

Rubinstein JT, Turner CW (2003) A novel acoustic simulation of cochlear implant hearing: effects of temporal fine structure. First. Int. IEEE. EMBS. Conf. Neural. Eng. IEEE Press, 142-145

SAberi K, Green D (1997) Evaluation of maximum-likelihood estimators in nonintensive auditory psychophysics. Percept Psychophys 59:867-876

Saji AA, Litvak LM, Spahr AJ, Eddins DA (2009) Spectral modulation detection and vowel and consonant identifications in cochlear implant listeners. J Acoust Soc Am 126:955-958

Shannon RV, Zeng FG, Kamath V, Wygonski J et al (1995) Speech recognition with primarily temporal cues. Science 270:303304

Shelton B, ScArrow I (1984) 2-Alternative versus 3-alternative procedures for threshold estimation. Percept Psychophys 35:385-392

Shelton B, Picardi M, Green D (1982) Comparison of 3 adaptive psychophysical procedures. J Acoust Soc Am 71:1527-1533

Spoor A, Timmer F, Odenthal DW (1969) The evoked auditory response to intensity modulated and frequency modulated tones and tone bursts. Int Audiol 8:410-415 
Supin A, Popov VV, Milekhina ON, Tarakanov MB (1994) Frequency resolving power measured by rippled noise. Hear Res 78:31-40

Turner CW, Gantz BJ, Vidal C, Behrens A et al (2004) Speech recognition in noise for cochlear implant listeners: benefits of residual acoustic hearing. J Acoust Soc Am 115:1729-1735

Wojtczak M, Donaldson GS, Viemeister NF (2003) Intensity discrimination and increment detection in cochlear-implant users. J Acoust Soc Am 114(1):396-407
Won JH, Drennan WR, Rubinstein JT (2007) Spectral-ripple resolution correlates with speech reception in noise in cochlear implant users. J Assoc Res Otolaryngol 8:384-392

Won JH, Schimmel SM, Drennan WR, Souza PE et al (2008) Improving performance in noise for hearing aids and cochlear implants using coherent modulation filtering. Hear Res 239:1-11

Won JH, Drennan WR, Kang RS, Rubinstein JT (2010) Psychoacoustic abilities associated with music perception in cochlear implant users. Ear Hear 31:796-805 